\title{
Biosimilars Revolution
}

\author{
M. Salah Eldin Abdel Baky \\ Professor of Internal Medicine, Rheumatology and Immunology \\ Department of Internal Medicine, Division of Rheumatology, Ain Shams University; Egypt
}

\begin{abstract}
A biosimilar is an officially regulated and approved copy of an originator biologic therapy. Improved affordability and consequent wider patient access compared with biologics are a significant appeal of biosimilars.

Regulatory guidelines for biosimilar development and approval are rigorous and undergoing constant refinement. The process of licensing approval for all biosimilars requires demonstration of comparability in quality, efficacy, and safety between the biosimilar and reference (originator) product, which is undertaken in a stepwise procedure of nonclinical and clinical evaluation.
\end{abstract}

Biologics, both biosimilars and their corresponding reference products, are complex molecules produced by biotechnology in living systems. The development of biologics involves multiple levels of highly controlled manufacturing processes, combined with pre-clinical structural, functional, and biological assessments, as well as clinical efficacy and safety, including immunogenicity, analyses. In addition, to ensure a high degree of similarity, a biosimilar must undergo a comparability exercise at every step of its development, as outlined by regulatory agencies, to demonstrate that potential differences from the reference product are not clinically meaningful with regard to quality, safety, and efficacy [European Medicines Agency (EMA)] or safety, purity, and potency [US Food and Drug Administration (FDA)]. ${ }^{2}$

Major advances in biosimilars have occurred in the last three months, ending with the FDA recent approval of Inflectra (generically referred to as infliximab dyyb).

The U.S. Food and Drug Administration's approval of the biosimilar Inflectra (infliximab-dyyb) was met with great interest from physicians and patient advocacy groups who expressed concerns about safety, interchangeability and costs.

Inflectra (infliximab-dyyb), which is a biosimilar to infliximab (Remicade), was FDA approved on April 5 for patients with moderate to severe rheumatoid arthritis, Crohn's disease, ulcerative colitis, ankylosing spondylitis, psoriatic arthritis and plaque psoriasis, which are the approved indications for Remicade.

For Correspondence: e-mail: Salahym50@Yahoo.com
Inflectra's approval comes after a rigorous review process that included a series of clinical trials in rheumatoid arthritis and ankylosing spondylitis (AS). The trials demonstrated that it was a safe and effective biosimilar to Remicade. However, the FDA was quick to highlight that it was not approved as an interchangeable product and instead, is merely an option that could potentially expand access to treatment for patients. ${ }^{3}$

The American College of Rheumatology (ACR) applauded the decision citing a potential cost savings for patients, but they also expressed concerns over the need for distinct names and transparent labeling for all biosimilar products to ensure correct prescribing and dispensing, post-marketing surveillance, prescriber confidence and enhanced market uptake. ${ }^{4}$

IMS Institute for Healthcare Informatics has reported that the introduction of biosimilars in the US and Europe's five top markets could result in a $\$ 110$ billion ( $\$ 98$ billion euros) savings by $2020 .^{5}$

2014, worldwide sales of Remicade (infliximab) topped $\$ 9.88$ billion, with more than $\$ 4$ billion in U.S. sales. The biosimilar of Remicade (Remsima, Inflectra) is approved by the European Medicines Agency (EMA) and is used in more than 60 countries.

There are over 700 biosimilar products in preclinical and clinical trials. To date, 30 applications for biosimilar products have been evaluated in the European Union and 22 were approved across six classes. In the United States, the FDA reports that 59 proposed biosimilars for 18 different reference products are enrolled in its Biosimilar Product Development Program. ${ }^{6}$

In February 2016, the British Society of Gastroenterology issued a guidance document about switching patients from infliximab to (Remsima). The BSG concluded that there is sufficient data from observational studies to show that safety and clinical efficacy of (Remsima) are comparable to the originator drug, with similar immunogenicity, and that switching from Remicade to (Remsima) is also safe and effective. Patients should be switched at the same dose and interval. Automatic substitution at the pharmacy level without consulting the prescriber was stated to be inappropriate. ${ }^{7}$

Regulations for registration of biological products have been recently implemented in Egypt in 2009 through the Minister Decree 297/2009 adopting 
guidelines for submission of registration dossier based on full data (quality, preclinical and clinical).

Egyptian guidelines had been developed in order to facilitate the registration of biosimilar products in Egypt through an abbreviated pathway. These guidelines apply to well characterize Biological Medicinal Products developed by means of biotechnology (including recombinant DNA technology). The difference between the term generic used for description of copies for reference pharmaceutical product and the term biosimilar used to describe the similar versions of reference biological product should be clearly understood. ${ }^{8}$

Biosimilars continue to command a great deal of attention given the promise of significant cost savings and potentially wider use for our patients who are in bad need for these medications.

\section{REFERENCES}

1. Biosimilars: A Multidisciplinary Perspective. Khraishi M, et al. Clin Ther 2016 Mar 14

2. Development of biosimilars. Al-Sabbagh A, et al. Semin Arthritis Rheum. 2016; 45 (5 Suppl): S11-8.

3. Clinical and regulatory perspectives on biosimilar therapies and intended copies of biologics in rheumatology. Mysler E, et al. Rheumatol Int. 2016 Feb 27

4. http://www.rheumatology.org/

5. http://buff.ly/1qqvIn

6. Critical Points to Consider about Biosimilars. Amy Rhodes; The Rheumatology Network 3/16/16

7. http://buff.ly/23wpOad

8. http://www.eda.mohealth.gov.eg 